\title{
Screening for Psoriatic Arthritis in People with Psoriasis
}

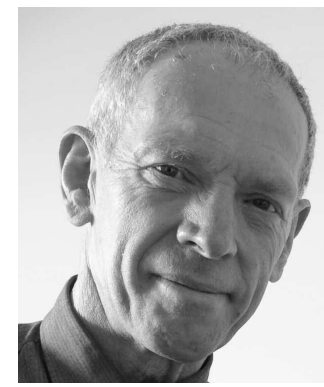

Psoriasis is the best biomarker for disease that we have in rheumatology. Seventy percent of people who develop psoriatic arthritis (PsA) will have psoriasis at presentation. The psoriasis may be "hidden" and may deceive the assessing physician, but generally the skin disease is known to the patient, and is obvious to the observer. So, given this strong association, can we predict those people with psoriasis who will go on to develop PsA? Some time ago it was reported that certain phenotypes of psoriasis are associated with the development of psoriatic arthritis: these include psoriasis of the nails, psoriasis of the scalp, and flexural psoriasis ${ }^{1}$. It is of note that these are all areas that are "hidden" to the examining physician - unless we look, we will never see. Refer to the article of Gorter, et al, who "sent" patients with hidden psoriasis to rheumatologists and fewer than $40 \%$ examined these hidden areas. And let us not forget the subclinical disease $^{2}$. Gisondi, et al were the first to demonstrate subclinical enthesopathy in patients with psoriasis ${ }^{3}$, and these abnormalities have also been found in other disorders, such as inflammatory bowel disease. However, the exact significance of these abnormalities remains unclear - a well-controlled longitudinal study in asymptomatic patients with psoriasis with (and without) such abnormalities is required to answer that question.

Of more importance perhaps is the number of patients who have already developed PsA but remain undiagnosed. Community surveys put this figure at about $15 \%$, and the equivalent figure in secondary care is around $30 \% 4,5$. This is, on the face of it, astonishing, and leads to speculation that this is not major disease but minor forms of enthesitis and oligoarthritis that are not affecting the patient. Sadly, this is not the case: In the community survey carried out in the UK, patients newly "discovered" had a mean duration of arthritis symptoms of 20.3 years, a mean swollen joint count of 3.9, a mean Health Assessment Questionnaire score of 0.72, and a mean Psoriasis Area and Severity Index score of $2.1^{4}$. Ten patients had clinical enthesitis but only 1 patient had dactylitis. In secondary care, in newly diagnosed cases, the mean swollen joint count was 4.1 , and the mean tender joint $9.0^{5}$.
How can this situation have happened? The answer requires further study, but we can speculate, in secondary care at least, that patients do not think it appropriate to tell their dermatology specialist about their articular symptoms. They may well do so in primary care; however, it must be that they are misdiagnosed, perhaps as some other arthropathy such as osteoarthritis or fibromyalgia. Certainly more education is needed to remind the primary care physician about the association between arthritis and psoriasis. From a secondary care point of view, educational initiatives are already under way, with dermatology publications highlighting the association ${ }^{6}$, and educational initiatives for a combined dermatology/rheumatology audience now becoming increasingly available under the auspices of the Group for the Research and Assessment of Psoriatic Arthritis (GRAPPA: see grappanetwork.org for further details). Such combined meetings emphasize that we are dealing with one disease (psoriatic disease) and that each specialty has something to learn from the other. The sentiments are not solely one-sided, as rheumatologists are encouraged to take account of the skin in making treatment decisions for their patients. In an ideal world this would happen, with the patient consulting both dermatologist and rheumatologist jointly.

Is it reasonable to ask a dermatologist to assess the musculoskeletal system? Psoriasis is the dermatological manifestation of psoriatic disease, which may have a significant musculoskeletal component, but may also include pathology at other sites, such as the eye and gut, and can be associated with significant cardiovascular morbidity. Physicians are now encouraged to consider this disease complex and to ensure that appropriate monitoring, and if necessary treatment, of all the manifestations takes place. Really, the situation is no different from that of a connective tissue disease, such as systemic lupus erythematosus or systemic sclerosis, which can affect multiple organs and may be managed by a number of specialties.

The CASPAR criteria enabled uniformity in disease classification for PsA. However, dermatologists find it difficult to apply these criteria to their patients with articular symp-

See Validation of ToPAS 2, page 841

Personal non-commercial use only. The Journal of Rheumatology Copyright (c) 2015. All rights reserved 
toms because the elements of the stem (inflammatory musculoskeletal disease: arthritis, enthesitis, or spondylitis) remain undefined. GRAPPA is currently trying to rectify this; meanwhile, what can dermatologists do to identify the patients with articular symptoms who need to be seen by their rheumatologist colleague? There are 2 approaches. First, some key clinical features that can be reliably identified would help in the selection process. The ability to recognize a swollen joint, enthesitis, or dactylitis, an everyday skill for a rheumatologist, may exceed the threshold of competence for a dermatologist, although 1 study found good interrater reliability by dermatologists for tender, but not swollen, joint counts in patients with $\mathrm{PsA}^{7}$.

The second approach does not rely on the skill or the motivation of the dermatologist and can be carried out by clinic staff. This is the screening questionnaire. Since the first of these (developed in Canada in $2000^{8}$ ), a number of such tools have been introduced ${ }^{9,10,11}$. The basic premise is the same: ask simple questions about inflammatory musculoskeletal symptoms with the intention of identifying significant, and relevant, symptoms, using a cutoff score to distinguish relevant cases. How do these tools perform in practice? Not surprisingly, the screening tools performed better in their development cohort than when tested on an independent cohort. The CONTEST study compared the Psoriatic Arthritis Screening Evaluation (PASE), Toronto Psoriatic Arthritis Screen (ToPAS), and the Psoriatic arthritis Epidemiology Screening Tool (PEST) in a head-to-head study in UK secondary care ${ }^{12}$. Patients with a diagnosis of psoriasis (excluding cases of PsA) were invited to participate and complete each of the 3 screening tools, presented in random order - those scoring positive on any of these were invited to attend for a rheumatological examination. It should be noted that the design of this study did not allow an assessment of the true sensitivity (which was probably overestimated) or the true specificity (which was underestimated). Nevertheless, 3 interesting findings appeared. First, there was no significant difference between the tools, although the PEST had the highest area under the receiver operating curve. Second, the sensitivity, and in particular, the specificity were worse than found in development (the specificities were in the $30-40 \%$ range), and largely due to people with noninflammatory disease responding positively to the questionnaires. Third, the more questionnaires were answered positively, the more likely the patient was to have undiagnosed PsA, suggesting that perhaps certain key elements between the 3 tools were more discriminatory. Interestingly, a parallel study in Dublin found an entirely different result - low sensitivities and higher specificities, not finding much in the way of noninflammatory disease ${ }^{13}$. The Dublin group argued that the tools had low sensitivities because they were less likely to identify such aspects of PsA as axial disease and enthesitis.

In the current issue of The Journal, the latest version of the Toronto Psoriatic Arthritis Screening Tool (ToPAS 2) is described ${ }^{14}$. This tool has been modified to include more specific questions on axial disease, and images of skin, inflamed joints, and dactylitis have been included. In this validation phase the revised version performed well in the cohorts tested (patients with known PsA, patients with psoriasis without PsA, and healthy controls). In practice this revised tool should be more sensitive to the pure axial forms of the disease. The authors recognize the limitation of adopting a retrospective case-control type design, in particular with respect to answering questions involving the performance of this instrument in screening for PsA in an unselected psoriasis population. Presumably this is their next study, hopefully with other instruments for comparison. These studies are eagerly awaited, although, because of the heterogeneity of PsA phenotypes, a "perfect" screening tool based on symptoms will be difficult to achieve.

Finally, what role do these instruments play in the other group of patients mentioned above - those destined to develop PsA but who do not yet seem to have musculoskeletal symptoms? On the face of it this is a nonstarter: people without aches and pains will not score positively on these questionnaires. Yet how often do physicians in practice discover symptoms (and signs) that have been unrecognized (or ignored, or belittled) by patients? People may minimize symptoms and signs because of fear, or because they are thought to be just part of our everyday lot. Back pain, for example, in epidemiological surveys is extremely common yet very few patients consult their doctor about these pains. It is possible therefore that these instruments may "reveal" symptoms that the patients were not prepared to disclose to their physician or felt that they were not of any import.

A good proportion of patients with psoriasis also have PsA yet remain undiagnosed. Raising awareness of the association between psoriasis and arthritis, both in patients and physicians, should help reduce this anomaly in future. The use of screening questionnaires raises awareness if nothing else, and may even help to sort out those patients who have PsA as distinct from other arthropathies. At least it will alert the patient to talk to their physician about their musculoskeletal symptoms when in the past they may have thought that dermatologists were uninterested in anything but the skin. The concept of screening for other comorbidities, such as the metabolic syndrome, is next on the agenda.

PHILIP S. HELLIWELL, FRCP,

Leeds Institute of Rheumatic and Musculoskeletal Medicine, University of Leeds, Leeds, LS7 4SA, UK.

Address correspondence to Dr. P.S. Helliwell, NIHR Leeds Musculoskeletal Biomedical Research Unit, Leeds Institute for Rheumatology and Musculoskeletal Medicine, Chapel Allerton Hospital, Chapel Town Road, Leeds, LS7 4SA, UK. E-mail: p.helliwell@leeds.ac.uk.

\section{REFERENCES}

1. Wilson FC, Icen M, Crowson CS, McEvoy MT, Gabriel SE, Kremers HM. Incidence and clinical predictors of psoriatic arthritis

Personal non-commercial use only. The Journal of Rheumatology Copyright @ 2015 . All rights reserved. 
in patients with psoriasis: a population-based study. Arthritis Rheum 2009;61:233-9.

2. Gorter S, van der Heijde DM, van der Linden S, Houben H, Rethans JJ, Scherpbier AJ, et al. Psoriatic arthritis: performance of rheumatologists in daily practice. Ann Rheum Dis 2002;61:219-24.

3. Gisondi P, Tinazzi I, El Dalati G, Gallo M, Biasi D, Barbara LM, et al. Lower limb enthesopathy in patients with psoriasis without clinical signs of arthropathy: a hospital-based case-control study. Ann Rheum Dis 2008;67:26-30.

4. Ibrahim G, Waxman R, Helliwell PS. The prevalence of psoriatic arthritis in people with psoriasis. Arthritis Rheum 2009;61:1373-8.

5. Reich K, Kruger K, Mossner R, Augustin M. Epidemiology and clinical pattern of psoriatic arthritis in Germany: a prospective interdisciplinary epidemiological study of 1511 patients with plaque-type psoriasis. Br J Dermatol 2009;160:1040-7.

6. Warren RB, Helliwell PS, Chinoy H. Time for a 'joint' approach? Br J Dermatol 2013;168:683-4.

7. Chandran V, Gottlieb A, Cook RJ, Duffin KC, Garg A, Helliwell P, et al. International multicenter psoriasis and psoriatic arthritis reliability trial for the assessment of skin, joints, nails, and dactylitis. Arthritis Rheum 2009;61:1235-42.

8. Alenius GM, Stenberg B, Stenlund H, Lundblad M, Dahlqvist SR. Inflammatory joint manifestations are prevalent in psoriasis: prevalence study of joint and axial involvement in psoriatic patients, and evaluation of a psoriatic and arthritic questionnaire. J Rheumatol 2002;29:2577-82.
9. Husni ME, Meyer KH, Cohen DS, Mody E, Qureshi AA. The PASE questionnaire: pilot-testing a psoriatic arthritis screening and evaluation tool. J Am Acad Dermatol 2007;57:581-7.

10. Gladman DD, Schentag CT, Tom BD, Chandran V, Brockbank J, Rosen C, et al. Development and initial validation of a screening questionnaire for psoriatic arthritis: the Toronto Psoriatic Arthritis Screen (ToPAS). Ann Rheum Dis 2009;68:497-501.

11. Ibrahim GH, Buch MH, Lawson C, Waxman R, Helliwell PS. Evaluation of an existing screening tool for psoriatic arthritis in people with psoriasis and the development of a new instrument: the Psoriasis Epidemiology Screening Tool (PEST) questionnaire. Clin Exp Rheumatol 2009;27:469-74.

12. Coates LC, Aslam T, Al Balushi F, Burden AD, Burden-Teh E, Caperon AR, et al. Comparison of three screening tools to detect psoriatic arthritis in patients with psoriasis (CONTEST study). Br J Dermatol 2013;168:802-7.

13. Haroon M, Kirby B, FitzGerald O. High prevalence of psoriatic arthritis in patients with severe psoriasis with suboptimal performance of screening questionnaires. Ann Rheum Dis 2013;72:736-40.

14. Tom BD, Chandran V, Farewell VT, Rosen CF, Gladman DD. Validation of the Toronto Psoriatic Arthritis Screen, version 2 (ToPAS 2). J Rheumatol 2015;42:841-6.

J Rheumatol 2015;42:736-8; doi:10.3899/jrheum.150257 